\title{
Conociendo a la nueva clientela: una mirada al comportamiento del consumidor en Wal-Mart Supercenter en Shanghai
}

DOI: $10.32870 /$ mycp.v13i39.350

Clemente Hernández Rodríguez ${ }^{1}$

Francisco Javier Valderrey Villar ${ }^{2}$

\section{Resumen}

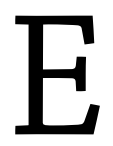
1 sector del comercio minorista en la República Popular de China se mantuvo sin cambios por décadas hasta la admisión del país a la Organización Mundial del Comercio en 2001 y la apertura del sector minorista en 2004, dando oportunidad a que cadenas extranjeras entraran al mercado. Wal-Mart y otros recién llegados de envergadura causaron inmediatamente un impacto en el sector minorista y una fuerte competencia entre las compañías en las diferentes capas de distribución. Esta investigación examina los patrones de compra de los clientes de un Wal-Mart Supercenter en Shanghai, una de las ciudades más dinámicas e importantes de China. Se usa un método de observación. La investigación encontró resultados interesantes en las principales preferencias del consumidor y la ruta más común seguida por el cliente en su experiencia de compra. Los resultados apuntan a un grupo de variables que deben tenerse en cuenta cuando se enfrentan decisiones de gestión.

Palabras clave: comportamiento del consumidor, Wal-Mart, China, experiencia de compra.

1. Profesor-Investigador del Tecnológico de Monterrey (ITESM), Campus Guadalajara.

2. Profesor-Investigador del Tecnológico de Monterrey (ITESM), campus León. ORCID http://orcid.org/0000-0002-7160-8836 


\section{Abstract}

The retail sector in the People's Republic of China remained basically unchanged for decades; only the admission of the country to the WTO in 2001 and the subsequent opening of the retail sector in 2004, created the opportunity for foreign chains to enter the market. Wal-Mart and other colossal newcomers immediately caused an impact on the retail sector and a strong competence among firms at different layers of distribution. This research examines the shopping patterns of the clients of a Wal-Mart supercenter in Shanghai through an observational method. The investigation found interesting results on the main preferences of the consumer and the most common route followed by the client on their entire shopping experience. The results point to a group of variables to be taken into consideration when facing managerial decisions.

Keywords: Consumer behavior, Wal-Mart, China, experiencia de compra, buying experience.

\section{Introducción}

El mercado minorista chino experimentó la entrada del concepto de hipermercado en los comienzos de la década de 1980. Como consecuencia de las reformas económicas de Deng Xiaoping y la apertura de China al mundo, ${ }^{3}$ un gran número de visitas de turistas extranjeros y chinos de ultramar crearon una necesidad que debía satisfacerse, y eso se realizó con los hipermercados. En la década de 1980 los hipermercados experimentaron un lento crecimiento (Mai y Zhao, 2004), pero a fines de la década de 1990 con la entrada de las compañías minoristas extranjeras con propiedad y participación parcial, el concepto se expandió mucho más rápido.

Para diciembre de 2004 China, siguiendo las reglas de la OMC, ${ }^{4}$ abrió completamente sus puertas a las compañías minoristas extranjeras, lo que significa "permiso para invertir independientemente en cualquier ciudad" (Chandler, 2005). La población de China compone una quinta parte de la población mundial, una gran oportunidad para las cadenas minoristas extranjeras; sin

3. Den Xiaoping es recordado como la mente maestra de las reformas económicas en la República Popular de China. Después del inicio de la década de 1980 sus reformas se enfocaron a la agricultura, industria, ciencia y defensa.

4. Entre 2001 y 2005 China cumplió con muchos de los requisitos impuestos como condición para pertenecer a la Organización Mundial del Comercio (OMC). 
embargo el consumidor chino es muy diferente al consumidor occidental y eso debe tomarse en cuenta. La guerra por la supremacía de supermercados ha comenzado; no obstante, los competidores tradicionales como los mercados de alimentos continúan desempeñando un papel importante.

La investigación realizada sobre el entorno económico de China se ha centrado en factores macroeconómicos, tales como análisis de riesgo país para inversionistas o información demográfica; pero la investigación que se centra principalmente en el consumidor chino sigue siendo insuficiente, a pesar del nuevo crecimiento en el ingreso disponible general. Por lo tanto, el siguiente estudio analiza los hábitos de consumo en Wal-Mart Supercenter de Shanghai. La zona de Shanghai cuenta con una gran densidad de población (la población estimada en 2007 excedió los 18 millones de personas), ocupa el primer lugar en importancia comercial, tiene los salarios promedio más altos en la República Popular de China (aproximadamente 39,004 yuanes por año), siendo así una de las áreas más interesantes para los inversionistas. ${ }^{5}$

Esta investigación, llevada a cabo en el verano de 2008, se realizó con el propósito de proporcionar nuevo conocimiento sobre la forma en que el consumidor chino compra, todo con la esperanza de proporcionar información valiosa para la toma de decisiones.

La principal hipótesis del estudio es que la mayoría de los consumidores compran principalmente comida cuando visitan los supermercados.

\section{Wal-Mart en China}

El primer Wal-Mart Supercenter localizado en Shanghai fue construido en concordancia a la importancia de la ciudad; como se ha señalado anteriormente, Shanghai está ubicada entre las principales zonas para actividades comerciales. Shanghai es una ciudad bajo la influencia de marcas e ideas extranjeras; de hecho, históricamente ha sido un puerto para cambiar divisas. Sin embargo, la influencia de la China tradicional es evidente. Por consiguiente, la ciudad proporciona un campo excepcional para investigar los patrones de

5. La gran zona de Shanhgái está entre una de las áreas más dinámicas a lo largo de la costa, con un continuo crecimiento en la zona del aeropuerto de Pudóng, y expandiéndose rápidamente hasta llegar a las pequeñas ciudades. Un gran número de proyectos de transporte ha favorecido la integración de importantes ciudades en la zona de influencia económica de Shanhgai (China Statistical Yearbook, NBSC, 2007). 
comportamiento del consumidor y las nuevas tendencias inducidas por las nuevas generaciones.

Wal-Mart es la compañía de minoristas más grande del mundo. Sin embargo, Wal-Mart no mantiene una posición dominante en la nación asiática. Varios minoristas locales siguen estando por delante, así como el gigante francés Carrefour. A pesar de eso, hay un esfuerzo importante de Wal-Mart para posicionarse como el número uno en el mercado de minoristas. Como sucede en todo el mundo, es difícil saber el último número de unidades de WalMart en el país. Recientemente abrió el supercentro número 100, opera en 55 ciudades y empleaba a más de 300,000 empleados, con una inversión global de aproximadamente dos billones de renmimbi (rmb) por año ${ }^{6}$ ( $\left.\mathrm{CBR}, 2005\right)$.

\section{Revisión de la literatura}

La revisión de trabajos anteriores de investigación se enfoca en el conjunto de las siguientes variables: precio, marca, calidad, factores demográficos y sus efectos en los consumidores y la publicidad.

\subsection{El precio}

La sociedad china creció con el concepto de que ser ahorrativo es una gran virtud; como consecuencia, el precio cotizado debe ser razonable. También, un bajo precio a menudo implica poca calidad. Cuando enfrenta una decisión entre dos productos similares que difieren mucho en precio, el comprador probablemente escogerá el más caro de los dos, ya que es posible que perciba el más económico como defectuoso (Gong, 2003). Un proverbio afirma la importancia de ser ahorrativo: "Nunca hagas una compra hasta que compares tres tiendas" (Dong y Gallup, 1995). Pagar más de lo que debería pagarse resulta vergonzoso, pero comprar un producto con una etiqueta de precio más bajo puede transmitir la idea de menor rango social. La sociedad no percibe la toma de riesgos como algo admirable, por lo que no es común que el consumidor asuma los riesgos cuando compra productos. Sin embargo, un estudio hecho por Mai y Zhao (2004) en China muestra que en el entorno

6. El renmimbi (o el dinero del pueblo), abreviado rmb, es la moneda china, mejor conocida en Occidente como el yuan. Al tiempo que la investigación fue realizada, un dólar estadounidense equivalía a $7 \mathrm{rmb}$. 
regular del supermercado los usuarios no suelen revisar los precios; después de todo, ya conocen sus preferencias y el precio esperado. Por lo tanto, se podría decir que estos clientes ya están familiarizados con el entorno de compra y tomarán decisiones basadas en factores diferentes al precio. El precio, entonces, se va a un segundo lugar en la importancia de los criterios (Schlevogt, 2000).

El segmento de edad es otro criterio importante relacionado a las actitudes hacia el precio. Está demostrado que quienes crecieron en la época Deng Xiaoping o un poco antes, a diferencia que las

El segmento joven, que considera la compra de productos más caros como muestra de éxito social y lujo, es, pues, más propenso a guiarse por los valores occidentales personas mayores a 65 años, están dispuestos a pagar precios más altos, cuando sea necesario (Anderson y He, 1998). El segmento joven, que considera la compra de productos más caros como muestra de éxito social y lujo, es, pues, más propenso a guiarse por los valores occidentales. Un claro ejemplo es cuánto cambia la percepción del precio entre las edades (Anderson y He, 1998).

Con respecto a las estrategias del precio, y cuando se enfrentan a ofertas de precios reducidos, los consumidores asumen que una empresa que vende artículos rebajados está haciendo "esfuerzos para deshacerse de un inventario que está por debajo de la media en calidad" (Anderson y He, 1998). Otras estrategias en los precios, como los cupones, se consideran adecuados sólo para los segmentos más bajos de la sociedad; por lo que un mercadólogo tiene que entender esos valores con el fin de evitar errores al diseñar sus estrategias de precio.

\subsection{La marca}

La marca es esencial para el nuevo consumidor chino. La entrada de marcas extranjeras después de años de productos nacionales sin diferenciar que ofrecían baja calidad, está dando paso a la fidelidad a la marca. Una marca que es considerada de buena reputación ganará la confianza del consumidor chino y será vista como una "garantía de calidad". La traducción una marca es muy importante por los muchos significados que la lengua mandarín o cantonesa 
pueden darle al nombre. Si la traducción es correcta, los minoristas tendrán resultados positivos en persuasión y en memoria. Los chinos están orientados a lo visual, así que las marcas deben desarrollar una buena imagen para el mercado; lo que le permitiría a una marca conocida y entendida ser más fácilmente reconocida y aceptada (Gong, 2003).

El origen de las marcas es muy importante ya que esto puede significar una compra o no, dependiendo del lugar donde fue fabricada (Bates, 1998). Cuando las marcas extranjeras entraron al mercado, el deseo por estas nuevas marcas creció, resultando en grandes ventas para sus productos. El efecto en el consumidor chino fue que le dio más importancia al nombre de la marca y al país de origen, como criterios importantes para la compra. En todo el mundo se acepta que las marcas conocidas tienen mucha presencia en la percepción del consumidor y éstas han servido para abrir mercado a sus connacionales.

La percepción de la marca es otro factor que influye amplia y positivamente en el comportamiento del consumidor. Esto significa que entre más notoria sea una marca, mayor será la probabilidad de que la compren con regularidad (Lin y Chang, 2003). La presentación del producto también es importante, ya que el empaque es ampliamente aceptado por los chinos. Por lo tanto, un producto occidental debe tener un empaque occidental y lo mismo pasa con los productos chinos. Sin embargo, las investigaciones muestran una mayor preferencia por la presentación de productos estilo occidental.

\subsection{La calidad}

El concepto de calidad ha evolucionado de ser un criterio sin significado a convertirse en una de las características clave al evaluar un producto para su compra (Schlevogt, 2000). La entrada de marcas y productos extranjeros también es una señal de bienvenida para los artículos de mayor calidad. Después, el punto de vista relacionado a la calidad pasó a ser fundamental en la mente del consumidor (Mai y Zhao, 2004). Los valores tradicionales han desempeñado un papel en la idea de calidad. Los chinos son conocidos por ser fatalistas: si un producto resulta ser de baja calidad, los defectos se pueden achacar al destino adverso. Por lo tanto, las garantías son de poca importancia para el chino, ya que esperan que sus productos siempre tengan un rendimiento consistente.

La búsqueda de información de un consumidor chino tiende a ser mucho más extensa que la de un consumidor occidental; como resultado, la proba- 
bilidad de elegir un producto de baja calidad es poca. Para ellos, una marca conocida evita tomar riesgos innecesarios (Li, Fuan et al., 2004). Una consecuencia de la importancia asignada a la lealtad a la marca es la sensación de ser engañado cuando el producto se convierte en defectuoso. Entonces será casi imposible recuperar la buena voluntad del comprador. La calidad percibida del producto debe ser siempre tomada en consideración (Lin y Chang, 2003).

\subsection{Factores demográficos y sus efectos en los consumidores}

La República Popular de China tiene la mayor población del mundo y, contrario a la creencia general, los ingresos y la cultura varían ampliamente. Los que acostumbran pensar en China como un ejemplo de igualdad y equidad social se pueden sorprender de visitar un país con gente de diferentes grupos étnicos, practicando diferentes religiones y que viven vidas realmente separadas. El porcentaje de personas que habitan en zonas rurales es mayor que el que vive en las zonas urbanas, con cerca de $67 \%$ de la población lejos de las ciudades. ${ }^{7}$ Esta parte de la sociedad, cerca de 800 millones de personas, por el momento no es de interés para los supermercados. Quienes habitan en las ciudades no son más de la tercera parte de la población total. No obstante, suman más de 400 millones de personas, viviendo en más de 33 ciudades con una población por encima de un millón de personas (Chandler, 2005). Los supermercados extranjeros se han enfocado a esas ciudades debido a las oportunidades de un mercado más próspero.

En comparación con sus contrapartes occidentales, los chinos son menos móviles. Además de las restricciones para viajar, un menor uso de automóviles significa que muchas personas utilizan bicicletas y transporte público. El tiempo de traslado al supermercado es más largo como resultado de esas restricciones. La mayoría de los clientes llegan al supermercado a pie, en bicicleta o en transporte público; también, tener que cargar sus compras da como resultado que se compren menos productos por visita (Mai y Zhao, 2004).

Una clara consecuencia es la importancia que adquieren la distancia y la conveniencia. Los comerciantes en China necesitan ampliar el canal de distribución, esperar un mayor número de visitas y preparase para una canasta de

7. Aunque esas cifras duras fueron tomadas del China Statistical Yearbook (NBSC, 2007), vale la pena mencionar que las zonas rurales de la República Popular de China están densamente pobladas y que el concepto de lo que es una ciudad difiere del de otros países. 
compras escueta. La población se compone principalmente por gente joven, con $70 \%$ de la población de entre 15 a 50 años, lo que indica una población atractiva para los mercadólogos. Los clientes entre la población en general son diferentes de los clientes más viejos, están separados por sus experiencias durante la revolución económica de Xiaoping. El ingreso promedio de la población urbana que visita los supermercados se puede separar en varios segmentos: no obstante, un grupo más grande estará ganando $800 \mathrm{rmb}$ mensualmente, mientras que un grupo significativo estará ganando más de 8,000 rmb por mes (Mai y Zhao, 2004). Esto debe tomarse en consideración cuando se crean estrategias de mercado (Anderson y He, 1998). El rol de la política de un hijo también crea una oportunidad de mercado para los supermercados, ya que una persona china (él o ella) tenderá a favorecer a su único hijo(a) para cumplir muchos de sus caprichos ${ }^{8}$ (Gimeno-Sarciada, 2003).

\subsection{La publicidad}

El concepto de publicidad a gran escala se introdujo por primera vez en China en 1979, cuando la Televisión Central China (cCTV) comenzó a utilizar la publicidad como una forma de promoción de varios productos locales. Esta emisión es considerada como el único medio masivo capaz de alcanzar casi todas las ciudades en China. Una gran variedad de temas tratados en los 16 canales de la CCTV han creado un creciente interés entre los ciudadanos chinos para ver la TV, el tiempo promedio de sintonía es de tres horas al día (Madden, 2006). La entrada de publicidad extranjera ha sido un éxito desde los años noventa, después de una serie de errores en los años ochenta, causados principalmente por publicidad que no fue ajustada a nivel local. Como resultado de los anuncios orientados al mercado, la población total ha dirigido su interés a ver lo que está disponible, con especial ansia por los productos extranjeros. El sistema político hábilmente utiliza los medios televisivos para favorecer sus políticas y para crear confianza en el consumidor chino. Ahora la televisión proporciona una herramienta importante para las marcas que desean entrar en el mercado.

8. La política de un hijo es impuesta por el Ministerio. Comenzó en los años ochenta con el objetivo de controlar el crecimiento de la tasa de población. A pesar de muchos esfuerzos por parte de las autoridades, la política no tuvo gran impacto en las zonas rurales; hoy en día, tener más de un hijo es una señal clara de realización y riqueza. 
Numerosos aspectos culturales deberían estar presentes en la mente de los mercadólogos antes de lanzar un nuevo anuncio, aunque la mente colectivista de los chinos merezca la mayor atención. Incluso cuando las ideas occidentales se han convertido en un concepto aceptado por las generaciones jóvenes, sigue siendo una sociedad que tiene en alta consideración el concepto de pertenencia a un grupo grande y único. Otra característica importante es que el chino prefiere ver la publicidad que presenta los productos de una manera más realista; en consecuencia un mercadólogo debe evitar la exageración u otras maneras de presentar productos fuera de su uso diario. El sistema para compra de tiempo aire en la CCTV es sorprendente, ya que es administrado por la apertura de subastas. Las empresas que presentan una mayor oferta (Madden, 2006) pueden controlar el tiempo aire: el número de minutos al aire, el tiempo premium, o incluso ambos. Ésta es una puerta abierta para que las grandes empresas controlen la publicidad en televisión. Las estrategias correctas tienen que ser diseñada a fin de tomar ventajas de este sistema de subasta.

\section{El estudio}

\subsection{El propósito del estudio}

La información que surge de estudios pasados sobre los consumidores chinos se ha centrado principalmente en factores como la calidad, la publicidad y la importancia de la marca. Sin embargo, pocas investigaciones se han dirigido hacia el modo en que un consumidor compra. Concretamente, se ha descuidado la investigación básica acerca de la ruta que el consumidor toma en torno al supermercado, la influencia de la distribución de los supermercados y el impacto de los estantes de promoción y las acciones, entre muchas otras.

Este estudio se centró en la experiencia de compra, tal como se entiende por el camino que el consumidor sigue a través de toda la visita al supermercado. Otros factores también han sido tomados en consideración, tales como la elección de un producto, la preferencia por un determinado medio de pago, o la actitud hacia las promociones, entre otros. Estas son las variables estudiadas con el fin de proporcionar información que no se ha recopilado previamente. En sí, el alcance del estudio de investigación es sugerir nuevas líneas de estudio para los investigadores y mercadólogos por igual.

Del propósito declarado de las intenciones de la investigación, surgen las siguientes preguntas: 
1. ¿Cuál es la distribución de la tienda?

2. ¿Cuál es la ruta del cliente?

3. ¿Qué productos tienen más probabilidades de ser vendidos?

4. ¿Los clientes van a utilizar carrito de compras o cestas?

5. ¿Cuál es la actitud del cliente hacia los estantes promocionales?

\subsection{Limitaciones de la investigación}

Existen varias cadenas de supermercados en la ciudad de Shanghai, algunas de ellas de capital nacional y otras respaldadas en parte o completamente por inversión extranjera. El Wal-Mart Supercenter ha sido elegido debido a su modelo de negocio y su importancia internacional, así como por el ambicioso crecimiento experimentado en China continental. Por lo tanto la investigación estuvo centrada únicamente en el Wal- Mart Supercenter de Shanghai, debido al tiempo y las limitaciones del idioma. Otra limitación del estudio es el tamaño de la muestra (29 personas), debido a las dificultades del uso de métodos de observación en la República Popular de China.

\section{Metodología}

La principal metodología de la investigación fue la observación directa de los clientes durante la experiencia de compra. Un cuestionario (véase tabla 1) fue llenado dentro de Wal-Mart Supercenter, con datos relevantes para estudio. El cuestionario incluía información acerca de la actitud hacia las marcas, el impacto de las promociones de productos, formas de pago, entre otras, así como datos demográficos. Es una herramienta sencilla diseñada con el propósito de pasar inadvertidos por los compradores observados. La encuesta fue completada en el transcurso de un fin de semana (sábado y domingo) desde temprano por la mañana hasta la hora de cierre.

\subsection{La muestra}

Los individuos de la muestra comparten las siguientes características:

- Clientes al azar, sin un rango de edad, sin preferencias de género. El nivel social podría variar desde clase alta a media.

- El método de muestreo fue el conveniencia. 
Tabla 1

El Cuestionario de Investigación

Cuestionario de Investigación

\begin{tabular}{llll}
\hline Sexo & Masculino () & Femenino () & \\
\hline Edad & $<20()$ & $21-30()$ & $31-40()$ \\
& $41-40()$ & $51-60()$ & $>60()$ \\
\hline Ruta de compra & (seguimiento de la ruta) & \\
\hline ¿Qué herramientas de compra son utilizadas? & & \\
& Carrito () Canasta ( ) Ambas ( ) \\
& Ninguna () \\
\hline Perspectiva de compra & & \\
& Compra de todo () \\
& Deja algunos productos () \\
& Deja todo () \\
\hline
\end{tabular}

Actitud hacia los estantes promocionales

Sigue caminando ( )

Observa y continúa caminando ( )

Observa y toma una muestra ()

Cuando selecciona un producto el cliente observa que:

Productos a la vista ( )

Productos hasta arriba ()

Todos los productos ()

Cuando selecciona un producto en la repisa:

Observa los productos alrededor ( )

Observa y selecciona ()

Observa y no selecciona ()

Cuando elige marcas ¿cuál es el origen de éstas?

Extranjeras () Chinas () Ambas ()

Cuando necesita ayuda del personal

No pregunta por ayuda ( )

Pregunta una vez ()

Pregunta dos veces ()

Pregunta más de dos veces ()

El personal proporciona ayuda al cliente ()

Formas de pago

Crédito () Efectivo ()

Tiempo empleado en la tienda

0-30 min. ( ) 31min.-1hr. ( )

1-1:30 hr. () >1:30 hr. () 
El tamaño de la muestra incluye 29 observaciones dentro de Wal-Mart Supercenter.

\section{Análisis de la información}

Se analizó la información con un software ampliamente utilizado, mostrando estadísticas básicas y la tabulación cruzada entre diferentes variables. Sin embargo, la información fue limitada por la naturaleza observacional de la investigación de campo.

\subsection{Objetivos}

Los principales objetivos de esta investigación fueron:

- Determinar el perfil del consumidor (edad y género).

- Localizar cuál fue la sección más visitada de Wal-Mart.

- Buscar la ruta específica del cliente durante la experiencia de compra.

- Encontrar una preferencia por el uso de canasta o carritos de compra.

- Observar las reacciones de los clientes hacia los estantes promocionales.

- Identificar la influencia en el arreglo de los productos en el comportamiento de compra.

- Encontrar preferencias para marcas china o extranjeras.

- Descubrir si el cliente pregunta por asistencia durante la compra.

- Observar la forma de pago más elegida.

- Medir el tiempo empleado por los clientes en las instalaciones, específicamente en cada área.

\subsection{Resultados}

La principal hipótesis del estudio de campo que indica que la mayoría de los clientes compran principalmente comida cuando visitan los supermercados se demostró por medio del gran porcentaje de visitantes en torno a las áreas relacionadas con alimentos. El género demostró no tener un impacto importante en la asistencia al centro de compra.

La tabla 2 muestra la distribución por edad y la distribución de género y por relación. Es interesante ver que muchas parejas compran juntas; sin embargo, la información fue recogida a lo largo de un fin de semana, durante el cual puede cambiar este comportamiento particular. El rango de edad de las 
personas de 31 a 40 años tuvo mayor presencia, seguido de cerca por aquellos del rango entre 21 a 30 . Hubo clientes menores de 20 y mayores a 50 años, pero obviamente una minoría.

Tabla 2

Muestra estadística demográfica

\begin{tabular}{lcc}
\hline \multicolumn{1}{c}{ Variable } & Frecuencia & Porcentaje (\%) \\
\hline Masculino & Género & \\
Femenino & 13 & $44.83 \%$ \\
Total & 16 & $55.17 \%$ \\
Relaciones & 29 & \\
Pareja & & \\
Familia & 10 & $35.71 \%$ \\
Solos & 4 & $14.29 \%$ \\
Total & 14 & $50.00 \%$ \\
\hline & 28 & \\
\hline$<20$ & Edad & \\
$21-30$ & 3 & $10.34 \%$ \\
$31-40$ & 7 & $24.24 \%$ \\
$41-50$ & 8 & $27.59 \%$ \\
$51-60$ & 6 & $20.69 \%$ \\
$>61$ & 3 & $10.34 \%$ \\
Total & 2 & $6.90 \%$ \\
\hline
\end{tabular}

La tabla 3 explica cómo fue dividido el supermercado en diferentes áreas, a saber:

Los puntos de recolección de información estuvieron en varias zonas de poco interés para los compradores, como juguetes y deportes, o plásticos, cerámica de cocina y suministros, y el área de electrónicos con poco o ningún atractivo. Por mucho, la zona de comida es la parte más visitada del supermercado (véase tabla 4).

La tabla 4 muestra el número de clientes (bajo el rubro núm.): en primer lugar aquéllos que visitaron la zona, en segundo lugar aquéllos que no la visitaron, y en tercer lugar, el porcentaje relativo del total de la muestra. Zonas como farmacia, ropa de invierno y cuidado personal son atractivas para muchos de los compradores. 


\section{Tabla 3}

\section{Diferentes áreas dentro del Supercenter}

\begin{tabular}{ll}
\hline & Descripción \\
\hline Zona 1 & Cereales, frutas y vegetales, y pescado \\
Zona 2 & Carne, aves congeladas, comida cocinada \\
Zona 3 & Carne empacada, congelados, comida envasada \\
Area 4 & Pastelería \\
Zona 5 & Alimentos cocinados, embalaje \\
Zona 6 & Joyería, artículos para el hogar \\
Zona 7 & Juguetes, bicicletas, deportes, zapatos, gimnasio \\
Zona 8 & Artículos para el hogar, cD, DVD \\
Zona 9 & Electrónicos \\
Zona 10 & Ropa, óptica \\
Zona 11 & Ropa de invierno, cosméticos, farmacia \\
Zona 12 & Cuidado personal, artículos para limpieza del hogar \\
Zona 13 & Plástico, cerámica y suministros de cocina \\
Zona 14 & Empaque \\
Zona 15 & Cajeros \\
\hline
\end{tabular}

\section{Tabla 4}

Distribución de clientes por zona

\begin{tabular}{lrrc}
\hline Zona & Núm. & \% Válido & \% Perdido \\
\hline Zona 1 & 21 & $72.41 \%$ & $27.59 \%$ \\
Zona 2 & 14 & $48.28 \%$ & $51.72 \%$ \\
Zona 3 & 10 & $34.48 \%$ & $65.52 \%$ \\
Zona 4 & 10 & $34.48 \%$ & $65.52 \%$ \\
Zona 5 & 10 & $34.48 \%$ & $65.52 \%$ \\
Zona 6 & 4 & $13.79 \%$ & $86.21 \%$ \\
Zona 7 & 3 & $10.34 \%$ & $89.66 \%$ \\
Zona 8 & 3 & $10.34 \%$ & $89.66 \%$ \\
Zona 9 & 1 & $3.45 \%$ & $96.55 \%$ \\
Zona 10 & 4 & $13.79 \%$ & $86.21 \%$ \\
Zona 11 & 5 & $17.24 \%$ & $82.76 \%$ \\
Zona 12 & 5 & $17.24 \%$ & $82.76 \%$ \\
Zona 13 & 2 & $6.90 \%$ & $93.10 \%$ \\
Zona 14 & 6 & $20.69 \%$ & $79.31 \%$ \\
Zona 15 & 29 & $100.00 \%$ & $0.00 \%$ \\
\hline
\end{tabular}


Las zonas de carne y aves congeladas atraen muchos clientes. Los consumidores chinos prefieren comprar carne fresca, a veces incluso animales vivos en el supermercado. Las zonas de cuidado personal, o relacionados con el tema (zona 11, cosméticos, farmacia y ropa de invierno), y los productos para el cuidado personal (zona 12) también son atractivos para la gente de la tienda.

El concepto de belleza ahora yace en la influencia occidental, probablemente aún más en una tienda de mercado al detalle, como se muestra por la amplia gama de cosméticos y productos de cuidado personal. Antes del comunismo los adornos y productos para mejorar la belleza eran comunes en las mujeres; durante el liderazgo de Mao Zedong los cosméticos eran vistos como una forma de narcisismo y materialismo, en contra de los ideales comunistas.

Finalmente, después de las reformas de Deng Xiaoping, los valores tradicionales relacionados a la feminidad están incrementado el atractivo de productos para el cuidado personal. Incluso los hombres ahora están apreciando la moda, mucho más entre las generaciones más jóvenes.

De mayor importancia fue observar la experiencia total de compra (véase tabla 5).

Básicamente, la tabla 5 muestra a un consumidor chino con rasgos interesantes. A pesar del tamaño del Wal-Mart Supercenter, los compradores no pasan mucho tiempo en el supermercado. La mayor parte del tiempo, los clientes emplean menos de 30 minutos en la tienda, en muy pocos casos estuvieron más de una hora. El efectivo fue la forma de pago más frecuente, probablemente debido a que las tarjetas de crédito no han llegado a la población en general.

Otro hecho digno de ser mencionado fue la preferencia por hacer pequeñas compras. De hecho, los compradores no suelen comprar muchos artículos, o demasiado volumen, ya que la mayoría llega al supermercado a pie o en transporte público.

Los chinos compran más productos nacionales que extranjeros. Esto puede no representar una respuesta hostil a los productos no chinos; muchos productos fabricados localmente pueden satisfacer mejor las necesidades de los compradores locales. La cultura china conservadora y de evitar riesgos afecta muchas variables, tales como el porcentaje de aquellos dispuestos a tomar una muestra de los estantes promocionales: la cifra es tan baja como $6 \%$; sin embargo, un porcentaje significativo se detiene y observa hacia el estante promocional, lo cual significa que la promoción podrá al menos llamar la atención de alrededor de $58 \%$ de los clientes. 
Clemente Hernández Rodríguez y Francisco Javier Valderrey Villar

Perfil de compra de los compradores

\begin{tabular}{|c|c|c|}
\hline Variable & Frecuencia & Porentaje(\%) \\
\hline \multicolumn{3}{|l|}{ Tiempo de espera (minutos) } \\
\hline $0-30$ & 14 & $48.28 \%$ \\
\hline $31-60$ & 11 & $37.93 \%$ \\
\hline $61-90$ & 4 & $13.79 \%$ \\
\hline \multicolumn{3}{|l|}{ Formas de pago } \\
\hline Efectivo & 23 & $79.31 \%$ \\
\hline Tarjeta de crédito & 6 & $20.69 \%$ \\
\hline \multicolumn{3}{|l|}{ Selección de la marca } \\
\hline China & 15 & $51.72 \%$ \\
\hline Extranjera & 1 & $3.45 \%$ \\
\hline Ambas & 13 & $44.83 \%$ \\
\hline \multicolumn{3}{|l|}{ Herramienta de compra } \\
\hline Carrito & 13 & $44.83 \%$ \\
\hline Canasta & 11 & $37.93 \%$ \\
\hline Ambas & 2 & $6.90 \%$ \\
\hline Ninguna & 3 & $10.34 \%$ \\
\hline \multicolumn{3}{|l|}{ Perspectiva de compra } \\
\hline Compra de todo & 18 & $62.07 \%$ \\
\hline Deja algunos productos & 11 & $37.93 \%$ \\
\hline \multicolumn{3}{|l|}{ Estantes de promoción } \\
\hline Sigue caminando & 10 & $34.48 \%$ \\
\hline Decide y se va & 17 & $58.62 \%$ \\
\hline Decide y toma una muestra & 2 & $6.90 \%$ \\
\hline \multicolumn{3}{|l|}{ Los clientes observan } \\
\hline Productos de reojo & 10 & $34.48 \%$ \\
\hline $\begin{array}{l}\text { Productos de la parte } \\
\text { superior }\end{array}$ & 1 & $3.45 \%$ \\
\hline Todos los productos & 18 & $62.07 \%$ \\
\hline \multicolumn{3}{|l|}{ Forma de elección } \\
\hline Observa productos alrededor & 8 & $27.59 \%$ \\
\hline Observa y elige & 19 & $65.52 \%$ \\
\hline Observa y no elige & 2 & $6.90 \%$ \\
\hline
\end{tabular}


En cuanto a la selección del nivel del estante, un gran número de compradores observan los productos alrededor antes de seleccionar; por lo tanto se comportan de acuerdo a un imperativo cultural de la comparación de tres productos (o tres tiendas) antes de tomar la decisión final. Aunque el mayor porcentaje selecciona productos mucho más rápido, quizás debido a su familiaridad con los productos y marcas. Curiosamente, todos los grupos seleccionaron únicamente aquellos productos que más tarde compraron, rara vez regresaron productos a la estantería o dejaron los productos que no deseaban en la canasta o carrito de compra. Estos resultados concuerdan con los de Mai y Zhao (2004). En cuanto al impacto de la altura en que el producto se coloca en la plataforma, cabe mencionar que de las personas observadas sólo una vio los productos a un nivel de posición superior a su línea visual. La mayoría de la gente observó todos los productos de la misma categoría adyacentes entre sí.

Otra cuestión importante para la investigación fue determinar si el consumidor pide ayuda cuando compra. Los resultados señalan claramente el disgusto de los consumidores chinos hacia preguntar por ayuda; $82.8 \%$ de los clientes no preguntan por ningún tipo de ayuda, y únicamente $13.8 \%$ de los clientes observados preguntaron una vez mientras que únicamente una persona pregunto en dos ocasiones. Al cliente chino tampoco le gusta expresar su insatisfacción si un producto no cumple con sus expectativas o está defectuoso. Ese comportamiento puede ser causado por la evasión de ser percibido como inepto para hacer sus compras por sí solo, lo cual podría generar vergüenza.

Se hizo un análisis de tabulación cruzada a fin de ver la relación entre diferentes variables. La edad demostró ser importante en relación con la elección de marcas nacionales o extranjeras. Únicamente una persona en el rango de los cincuenta años compró ambas marcas, nacionales y extranjeras, los otros clientes también en los cincuenta o mayores no compraron productos hechos en el extranjero. Lo contrario se observó con los grupos más jóvenes, que parecen tener preferencia por marcas internacionales.

Otro análisis de tabulación cruzada que busca una relación entre la edad y las formas de pago halló que únicamente grupos de jóvenes utilizaron tarjetas de crédito. También fue interesante no encontrar ninguna relación entre género y tiempo empleado en las instalaciones. La revisión de la literatura sugiere que los consumidores chinos tienden a pasar más tiempo en las instalaciones 
donde se sienten a gusto. Sin embargo, cambios profundos en el estilo de vida pueden limitar el impacto de un escenario cómodo.

Una variable clave fue la única relacionada a la atención que se presta a los accesorios promocionales, debido a la posibilidad de influir en los compradores en la tienda. La tabla 6 muestra que no hay diferencia en el comportamiento hacia de los estantes promocionales entre las personas de diferentes edades. Más interesante aún es el hecho de que esos accesorios atraigan poco a los compradores.

\section{Tabla 6}

Edad y atención a estantes promocionales

\begin{tabular}{lcccc}
\hline \multicolumn{5}{c}{ Tabulación cruzada de edad-estante promocional } \\
\hline Edad & Sigue caminando & $\begin{array}{c}\text { Se mantiene } \\
\text { se va }\end{array}$ & $\begin{array}{c}\text { Se mantiene y toma } \\
\text { una muestra }\end{array}$ & Total \\
\hline$<20$ & 2 & 1 & 0 & 3 \\
$21-30$ & 4 & 3 & 0 & 7 \\
$31-40$ & 2 & 5 & 1 & 8 \\
$41-50$ & 4 & 2 & 0 & 6 \\
$51-60$ & 0 & 3 & 0 & 3 \\
$>61$ & 1 & 1 & 0 & 2 \\
Total & 13 & 15 & 1 & 29 \\
\hline
\end{tabular}

También fue de gran importancia entender cuánto tiempo pasan los compradores en cada zona. La tabla 7 presenta los resultados obtenidos de comparar el tiempo total empleado en el supermercado y las visitas hechas a cada sección y la secuencia que siguieron: los pocos que pasaron más de una hora en el Supercenter fueron principalmente los clientes que pasaron por las zonas de alimentos. Sin embargo, hubo clientes que emplearon más de una hora y pasaron por la zona de cuidado personal.

Un objetivo importante del estudio de campo fue encontrar la ruta preferida de los clientes una vez dentro de las instalaciones, si dicha ruta existiera. Se obtuvieron resultados específicos para cada área y se muestran en la figura 1 .

Estos resultados apoyan la idea que más clientes visitan el supermercado en búsqueda de alimentos o productos relacionados. Para la mayoría de los compradores, el área de frutas, vegetales, cereales y pescado, fueron su primera selección en todo el supermercado. Sólo para una persona ésta fue su tercera zona visitada. La zona de carne, aves congeladas y comida cocinada 
Una mirada al comportamiento del consumidor en Wal-Mart Supercenter en Shanghai

Tabla 7

Tiempo total empleado en las zonas relacionadas con alimentos

\begin{tabular}{clccc}
\hline \multicolumn{5}{c}{ Tiempo total empleado por un cliente en la tienda } \\
\hline Zona & Secuencia & 0-30 min. & 31 min.-1hr. & $1-11 / 2 \mathrm{hr}$. \\
\hline 1 & Primero & 10 & 8 & 2 \\
& Tercero & 0 & 1 & 0 \\
& Primero & 1 & 2 & 1 \\
& Segundo & 3 & 6 & 0 \\
& Quinto & 0 & 0 & 1 \\
& Segundo & 3 & 0 & 1 \\
& Tercero & 2 & 2 & 0 \\
& Cuarto & 0 & 0 & 1 \\
& Sexto & 0 & 0 & 1 \\
& Primero & 1 & 0 & 0 \\
& Segundo & 0 & 2 & 0 \\
& Tercero & 2 & 0 & 2 \\
& Cuarto & 0 & 2 & 0 \\
& Quinto & 0 & 0 & 1 \\
& Primero & 3 & 0 & 0 \\
& Segundo & 2 & 1 & 0 \\
& Tercero & 1 & 1 & 0 \\
& Quinto & 0 & 1 & 1 \\
& & & &
\end{tabular}

fue elegida mayoritariamente como la segunda selección. Sin embargo, un número considerable de los que visitó la zona lo hizo en primer lugar. Junto con otras zonas relacionadas a alimentos, la sección de pastelería experimentó un número importante de visitas. Para muchos, esta fue una tercera selección de la ruta de compra; aunque hubo visitantes que llegaron a esta zona en primero, segundo u otro momento.

La zona de alimentos cocinados también registró una mayor presencia de visitantes en comparación con otras partes de la tienda no relacionadas con alimentos. Sin embargo, no hubo un patrón de tránsito visible, a veces se recogió como la primera, segunda, cuarta o incluso quinta elección de los clientes. Como se dijo antes, la mayoría de los clientes chinos visitan mucho la zona de alimentos y artículos relacionados (véase tabla 8). 


\section{Figura 1}

Secuencia en el área visitada

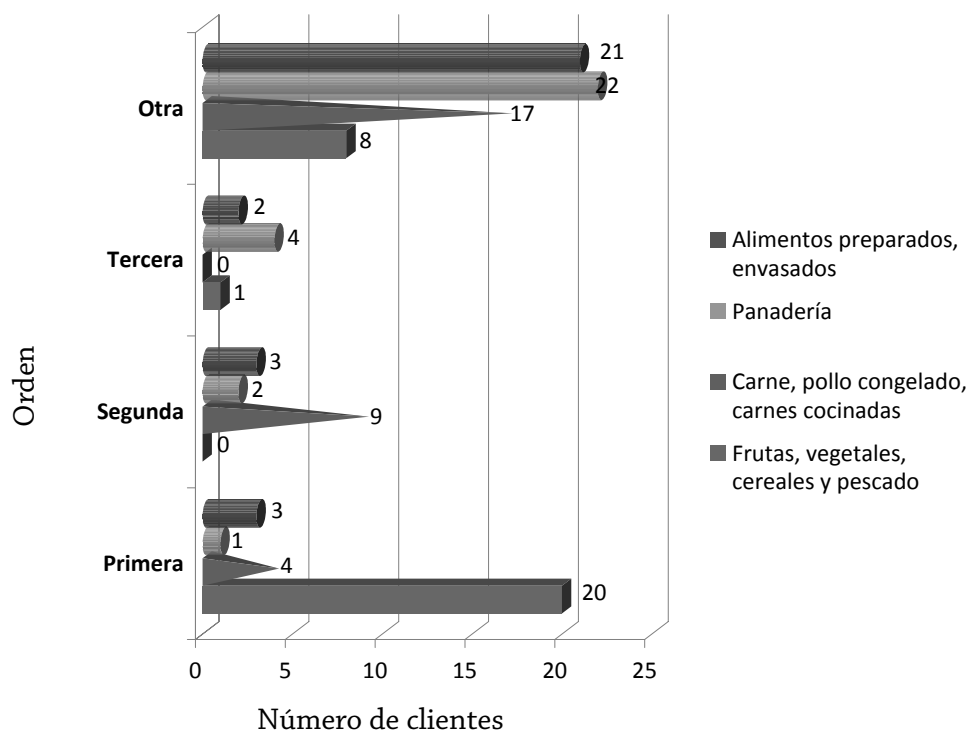

Tabla 8

Ruta preferida de compra

\begin{tabular}{ll}
\hline Secuencia & Zona \\
\hline Primera & Entrada \\
Segunda & Pescado, frutas y verduras \\
Tercera & Carne, aves congeladas, alimentos cocinados \\
Cuarta & Comida enlatada o pastelería \\
Quinta & Cuidado personal, ropa de invierno o panadería \\
Sexta & Varias zonas \\
Séptima & Joyería y juguetes \\
Octava & Paquetería \\
\hline
\end{tabular}

\section{Otras búsquedas}

Los supermercados de la República Popular de China probablemente presenten más diferencias que similitudes en comparación con los de Occidente. Las principales diferencias surgen del comportamiento del comprador. En 
un gran supermercado en China, uno debería esperar ver muchos empleados moviéndose a través de los pasillos, rara vez serviciales. Los clientes avanzan con rapidez, probablemente con una ruta preestablecida en mente. Las listas de compra no se ven por ningún lado, a excepción de en las manos de extranjeros; sólo este último grupo hace grandes compras y llevan los carritos llenos. Los lugareños comprarán productos para muy pocos días: resultado del estudio de campo puntual en un promedio de tres bolsas pequeñas de comestibles. Aunque en general se emplea poco tiempo en los locales comerciales, los compradores observan tenazmente y comparan antes de elegir el producto adecuado. Una vez que lo pensaron, el producto va a ser adquirido.

Incluso Wal- Mart Supercenter, un icono de la estandarización, será diferente en China. La pregunta, sin embargo, es si los hábitos y actitudes de los consumidores se adaptarán a las supertiendas o si es la corporación quien dicta la distribución y la apariencia de la tienda.

\section{Conclusiones}

Los compradores de supermercados en China tienen ciertas características que difieren de las culturas occidentales. Estas diferencias deben ser atendidas por los comercializadores a fin de reducir al mínimo el riesgo de fracaso una vez que entran al mercado chino. Es importante anotar que China es una nación con diferencias profundas. Los comercializadores necesitan ser extremadamente cuidadosos y buscar las claves para entender el medio ambiente desconocido. Los fabricantes necesitan entender las diferencias de la sociedad china antes de la promoción o incluso antes del diseño de los nombres de sus marcas. Al igual que con muchas de las tareas de mayor dificultad, la recompensa bien puede valer la pena.

\subsection{Factores demográficos}

Los factores demográficos desempeñan un papel importante en la forma en que los clientes hacen sus compras en China, en todos los aspectos del proceso de compra. Un gran porcentaje de la población continúa utilizando el transporte público o llega caminando a los supermercados, determinando la cantidad que compran y la frecuencia de asistencia. Los mercados tradicionales siguen teniendo un papel importante en China, lo que resulta una competencia para el mercado de frutas y verduras; algunas veces los mercados locales están cerca 
de los clientes, lo que les es conveniente. Contrario a muchas otras naciones, la gama de productos y una mejor comprensión de la clientela, constituyen una amenaza para las cadenas y los minoristas extranjeros. La distribución de la población tiene un efecto importante en dónde debe localizarse el supermercado, básicamente reduciéndose las oportunidades a ciertas zonas con un gran número de clientes y algunos ricos.

El sexo o la edad no parecen tener un papel importante en las compras del supermercado, aunque tomando en cuenta las limitaciones de este estudio, pero lo hacen en las preferencias de compra. El comerciante, por lo tanto, debe estar alerta a esos factores que desempeñan un papel en los factores de compra. La brecha entre los estratos socioeconómicos sigue siendo muy amplia: el desarrollo social en China continúa siendo altamente desbalanceado, con la mayoría de la población viviendo en zonas rurales, donde el ingreso disponible es claramente reducido. No obstante, el inequitativo desarrollo del país ha dado forma a nuevas ciudades industriales deseosas de ver mejores servicios y productos. Estos centros urbanos emergentes deberían tener una especial atención.

Un flujo de ideas extranjeras, marcas y perspectivas culturales están influyendo fuertemente la cultura ancestral, sobre todo entre las generaciones jóvenes. Sin embargo, la presencia del sabor y de las tradiciones chinas permanecerá por muchas décadas. Si se dirige correctamente, una mezcla de nuevos productos que atraigan a los chinos podría ser la tierra de oportunidades para los comercializadores internacionales. Entre esas novedades, el uso de la tarjeta de crédito parece estar en su infancia (aunque su uso está aumentando), mientras las nuevas herramientas de promoción necesitan ser concebidas para que sean llamativas entre los locales.

\subsection{Aspectos culturales}

La cultura china es adversa al riesgo, lo que afecta en gran medida el modo en que un cliente va a elegir dónde va a comprar y los productos que va a comprar. Sin embargo, hay una nueva generación con cambio de valores como el ser ahorrativo, o aquellos valores debido a la revolución, como la condición social. El nuevo consumidor está dispuesto a gastar más, aunque eso no signifique que sea más fácil que prueben nuevas marcas. El prestigio de una marca bien conocida, sin duda fomentará la lealtad de aquellos que no están dispuestos a arriesgarse con un producto nuevo. 
Cuando se habla de marcas extranjeras, un factor importante para los chinos es el origen del producto, ya que existen diferentes percepciones de cada país y ellas afectan la visión de los consumidores chinos hacia la marca. Aquellos países que son bien percibidos por los chinos tienen una ventaja competitiva sobre los países que son vistos como los fabricantes de productos inferiores. Los comercializadores de productos alimenticios extranjeros deben hacer un esfuerzo espacial para presentar sus productos naturales y frescos, como los que se encuentran en las tiendas locales. A los compradores les gusta tocar y sentir los productos antes de comprar.

En lo que respecta al uso de cupones de descuento y accesorios promocionales, debe buscar mejores maneras de atraer la atención del consumidor, ya que los resultados del estudio muestran poco impacto. El tiempo invertido en el supermercado es poco, en comparación a otros países; podría haber varias explicaciones pero es evidente que los compradores siguen una rutina, donde la compra de alimentos sigue siendo el principal objetivo para ir a un gran supermercado o centro de ventas al menudeo. Por lo tanto, los comercializadores podrían explorar diferentes maneras de ordenar los productos ofrecidos, mientras que se crea una atmósfera tentadora. En un país donde el niño es el rey, se debería prestar más atención a las necesidades de la familia y de los pequeños.

\section{Referencias}

Anderson, M. Patricia y Xiaohong He, "Price influence and age segments of Beijing consumers", Journal of Consumer Marketing, vol. 15, núm. 2, 1998, p. 152-160.

Bates, Colin, "The many China markets", The China Business Review, septiembre-octubre, 1998, pp. 26-32.

CBR (2005), "Profiles of Selected Beijing Retailers", The China Business Review, noviembre-diciembre, 2005, pp. 4-7.

Chandler, Clay (2005), "The great Wal-Mart of China", Fortune magazine, vol. 152, núm. 2, pp. 104-111.

Cui, Geng y Quimin Liu (2001), "Executive Insights: emerging market segments in a transitional economy: a study of urban consumers in China", Journal of International Marketing, vol. 9, núm. 1, pp. 84-106.

Dong, Li y Alec M., Gallup (1995), "In search of the Chinese consumer", The China Business Review, septiembre-octubre, 1995, pp. 19-22. 
Fuller, H. Frank, Jhon C. Beghin, Hu Dinghuan y Scott Rozelle (2004), “China's Dairy market: consumer demand survey and supply characteristics", Iowa State University, Center for Agricultural and Rural Development, Staff Report 04-SR 99, agosto.

Gimeno-Sarciada, Pilar y Verónica Shao (2003), "El mercado de panadería, bolletería y galletería en China”, Oficina Económica y Comercial de España en Shanghai, agosto.

Gong, Weng (2003), "Chinese consumer behavior: a cultural framework and implications", The journal of American Academy of Business, vol. 3.

Jing, Jin y Dai Yan (3 de agosto de 2005), "Too many hypermarkets in Shanghai", China Daily (edición para Norteamérica).

Kim, Jai-Ok, Sandra Forsythe, Gu Quingliang y Sook Jae Moon (2002), "Cross-cultural consumer values, needs and purchase behavior", Journal of Consumer Marketing, vol. 19, núm. 6.

Li, Fuan, Zhou Nan, Nicholls J.A.F, Zhuang Guijun y Carl Kranendonk (2004), "Internlinear or inscription? A comparative study of Chinese and American mall shoppers' behavior”, Journal of Consumer Marketing, pp. 51-61.

Li, Jun, Wang Yunyi y Nancy L. Cassill, "A comparative study on new retailing outlets in the Shanghai apparel market." Journal of Fashion Marketing and Management, vol. 8, núm. 2, 2004, pp. 166-175.

Lin, M. Y. y L. H. Chang (2003), "Determinants of habitual behavior for national and leading brands in China", Journal of product \& Brand Management, vol. 12, núm. 2, pp. 94-107.

Lu, Le e Ilan Alon (2004), "Analysis of the Changing Trends in Attitudes and Values of the Chinese: The Case of Shanghai's Young \& Educated", Journal of International and Area Studies, vol. 11, núm. 2, pp. 67-88.

Madden, Normandy (2006), "How China buys and sells TV", Advertising Age (Midwest region), vol. 77, núm. 19, p. 26.

Mai, Li-Wei y Hui Zhao (2004), "The characteristics of supermarket shoppers in Beijing", International journal of retail \& distribution management, vol. 32, núm. 1, pp. 56-62.

McHardy, Reid David y Jhon Walsh (2003), "Market entry decisions in China", Thunderbird International Business Review”, vol. 45, núm. 3, pp. 289-306. McNeal, U. James y Chyon- Hwa Yeh (2003), "Consumer behavior of Chinese children: 1995-2002”, Journal of consumer marketing, vol. 20, núm. 6, pp. 542-554. 
National Bureau of Statistics of China (NBSC), China Statistical Yearbook, China Statistics Press, octubre, 2007.

Sammuel, S. Nicholas, Li Elton y Heath McDonald (1996), “The purchasing behavior of Shanghai buyers of processed food and beverage products: implications for research on retail management", International Journal of Retail \& Distribution Management, vol. 24, núm. 4, pp. 20-28.

Schlevogt, Kai-Alexander, "The branding revolution in China", The China Business Review, mayo-junio, 2000, pp. 52-57.

SinoCast China Business Daily News, "Wal-Mart profits in Chinese market", Londres, 10 de agosto de 2004, p. 1.

Sun, Tao y Guohua Wu (2004), "Consumption patterns of Chinese urban and rural consumers", Journal of Consumer Marketing, vol. 21, núm. 4, pp. 245-253.

Taylor, Robert, “China's Consumer Revolution: Distribution reform, foreign investment and the impact of WTO", Asian Business \& Management, vol. 2, núm. 2, agosto, 2003, pp. 187-201.

Wang, Lu Cheng y Zhen Xiong Chen (2004), "Consumer ethnocentrism and willingness to buy domestic products in a developing country setting: testing moderating effects", Journal of Consumer Marketing, vol. 21, núm. 6, pp. 391-400.

Welcome to Wal-Mart China!, en: www.wal-martchina.com/english/index.htm, fecha de consulta: 06/10/ 2008.

Wong, Grace Khei-Mie y Lu Yu (2002), "Income and social inequality in China: impact on consumption and shopping patterns", International Journal of Social Economics, vol. 29, núm. 5, pp. 370-384.

Wu, Yanrui (1997), "Wealth and spending patterns in China", International Journal of Social Economics, vol. 24, núm. 7, pp. 1007-1022.

Zhang, Lao (16 de diciembre de 2002), "Consumers crowd modern stores", China Daily (edición para Norteamérica), p. 5. 